\title{
An Integrated Design Methodology
}

\author{
T. Freiheit, S. S. Park, V. Giuliani \\ Department of Mechanical and Manufacturing Engineering \\ University of Calgary \\ 2500 University Drive NW, Calgary, Alberta, T2N 1N4 \\ tfreihei@ucalgary.ca
}

\begin{abstract}
Global markets demand quick product development that is simultaneously cost-effective and meets stakeholder needs. Many tools and design methodologies have been developed that address individual aspects of the design problem, such as Axiomatic Design, Design for Manufacture, Life Cycle Design, etc. However, competitive viability can be put at risk when a product fails to achieve all customer, business, manufacturing, and regulatory requirements. To deliver all design requirements, an efficient integrated design methodology is required. This paper proposes a design approach which integrates previously developed design tools to economically achieve essential design objectives, within a framework that facilitates a rapid design process.
\end{abstract}

\section{Introduction}

Fast changing global markets demand that manufacturers quickly develop products that are simultaneously cost-effective and meet a variety of stakeholder desires and needs. The pressures of global competition, rapid change, and short product life cycles, make the development of new design methodologies important to manufactures. To survive in the hyper competitive environment of today's "knowledge based society," innovative product design is essential. In addition, designers must be careful of separating aesthetics and engineering, which may lead to unappealing or difficult to manufacture products.

One of the greatest obstacles in the realm of design is lack of meaningful measures. Many tools and design methodologies have been developed that address individual aspects of the design problem, such as Axiomatic Design, Design for Manufacture, Environmental Life Cycle Design, etc. However, competitive viability can be put at risk by global competition when a product fails to achieve all customer, business, manufacturing, and regulatory requirements. To be confident that a product or service has delivered all design requirements, an efficient integrated design methodology is required that can ensure an effective solution of the design tasks. This also includes coming up with inventive designs by creating novel and useful ideas $[1,2]$.

Making the design process into a scientific or mathematical pedagogy is especially important for young designers and for new designs. Most design processes are based on previous designs and are limited to evolutionary improvements in the design process. To leap forward often requires a paradigm shift. Three current design paradigms are multidisciplinary integrated design, adaptive design, and development of information technology.

The multidisciplinary integrated design approach, i.e. concurrent engineering, considers the life cycle of the product and uses a team based strategy to achieve good design and shorten development time [3]. Adaptive design concepts, i.e. reconfigurable or modular approaches, are used to increase flexibility of the product [4]. Information technology has been extensively leveraged to manage the design process, with tools such as the internet providing easy access to information. The greatest strength of these paradigms may be their influence on the culture of design, but they also have weaknesses, such as limited tools to gain insight into design requirements, as well as most existing processes do not integrate functional requirements with cost [5].

This paper proposes a design approach which integrates design tools to measure and achieve good technical and economic designs, and insure achievement of essential design objectives, all within a framework that facilitates a rapid design process. This framework is being developed to integrate and utilize all of the excellent design analysis and synthesis tools that have been developed. It is not intended to replace these tools. The objectives of this integrated design methodology are:

a. Integrate the requirements and constraints, quality, and economic aspects of design. 
b. Identify dependent functional requirements and design parameters.

c. Resolve conflict between different design aspects to provide an overall better design.

The main contribution of this paper is the application of mathematical tools to facilitate the attainment of these objectives. The organization of the paper follows: Section 2 outlines the integrated design framework with three design activities and the design process. Section 3 formulates the design problem through an enhanced design methodology by using Axiomatic Design and the House of Quality. Section 4 introduces a desk lamp example. Section 5 analyzes a weighting matrix through statistical tools to minimize information. Section 6 discusses other issues such as conflict resolution and fine tuning. The paper then concludes with recommendations for future work.

\section{Integrated design framework}

"Good design is about giving customers what they want. Great design is about giving customers what they didn't expect" Tom Peters [6]

A framework for integrating the design process was initiated by categorizing the design problem into three overlapping design activities of Quality, Economics, and Requirements (Figure 1). The Quality task is essentially one of measuring the excellence of the design. It includes evaluation of the design for simplicity, independence, adaptability, functionality, and robustness. The Economic task addresses issues related to cost in materials, labor, and time, as well as business strategic goals. The Requirements task addresses the achievement of design objectives through standardized tools and methodologies for individual aspects of the design problem, e.g. performance, safety, environmental, and aesthetic issues. To achieve an overall good design, all three of these tasks are of equal importance and the design must take advantage of complimentary and resolve conflicting solutions to the design problem.

The analysis and deduction of optimum designs based on these three activities will facilitates the delivery of a design that has the potential to exceed customer expectations. The integrated design methodology provides a basis for exceeding customer expectations by providing a framework to utilize most of the excellent design tools that have been developed to consistently and quickly deliver a cost-effective, good design. The methodology, outlined in Figure 2, begins with a statement of the design problem, and then systematically works through synthesis, formulation, and analysis, with iterative loops. In this study, we focus our efforts in the dotted section to aid the design process.

As noted by most who have formulated design methodologies, it is critical that the design problem is

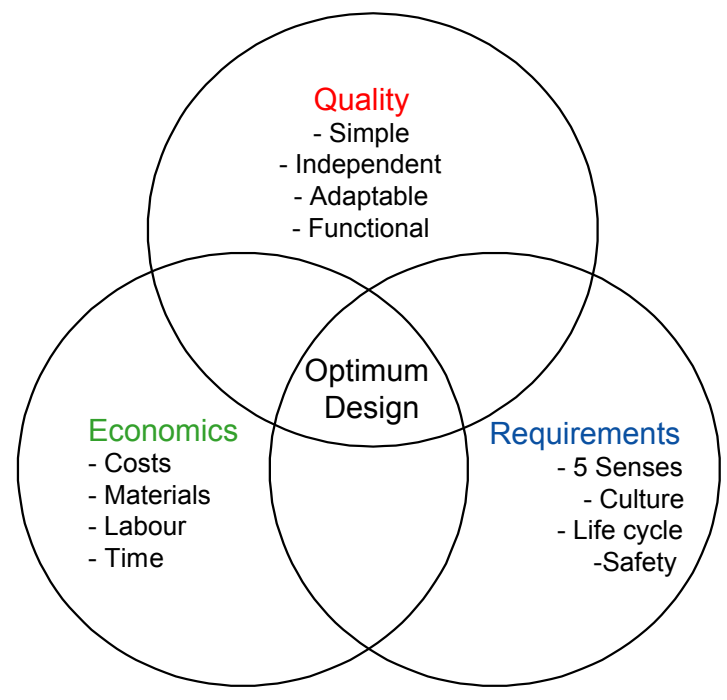

Figure 1. Integrated Design Activities

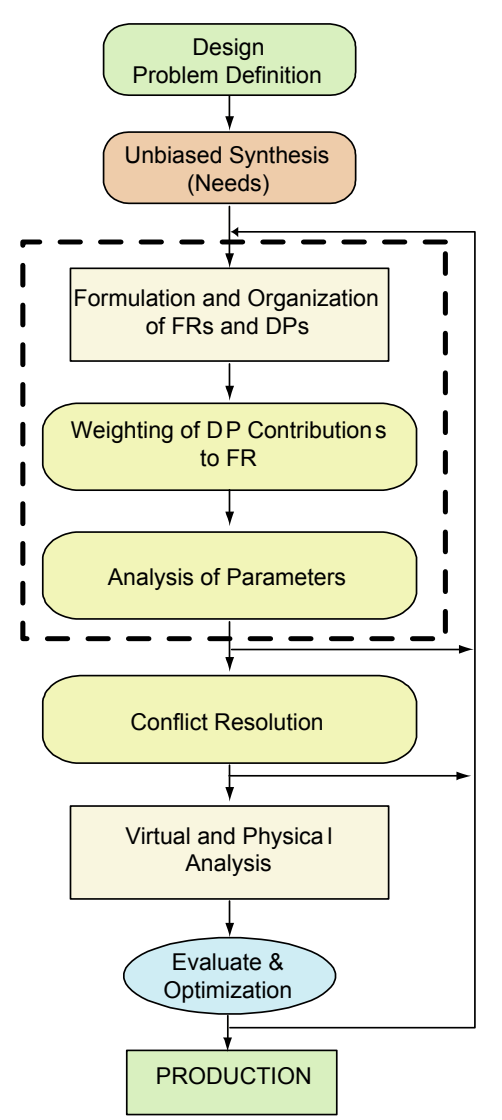

Figure 2. Integrated Design Process 
well formulated. Whereas in practice the design problem may be revisited and revised as design information is generated, this methodology starts with a well formulated problem definition to which an unbiased synthesis is applied. From the unbiased synthesis, a list of design constraints (requirements) and criteria (desires) is generated. From this, the design problem is reformulated as a set of sub-problems or functional requirements and a set of design parameters. Coming up with the functional requirements and design parameters can be very challenging. Out of the box thinking is very beneficial, which is why unbiased synthesis is so important.

The design is then analyzed for redundancy, robustness, and conflicts. When conflicts are found, the design is examined to find and evaluate compromises. Finally, detailed analysis and physical prototyping is performed and evaluated. In summary, the overall process is similar to the familiar 'needs definition, concept generation, concept selection, design embodiment, and prototype' process, where integration of the three design activities primarily occurs in the formulation of functional requirements and design parameters.

\subsection{Unbiased Synthesis}

Unbiased synthesis of imaginative ideas is essential part of the integrated design approach. Creativity is a driving force in good design and economic success. Whereas necessity may be the mother of invention, as Plato said in the Republic, unfortunately it tends to be neither systematic nor necessarily optimal. Like evolution, the solution of a problem by the expedient means of necessity tends to produce a solution that is only 'good enough'. Today's competitive economy demands solutions that go beyond 'good enough' to 'customer delight'. Today's customers are also equipped with a sea of information, such as the World Wide Web, and thus can easily avoid products that don't meet their exacting wants and needs. Several examples of tools for stimulating design creativity are using inversion or contradiction to come up with incremental or radical designs (TRIZ [7]), examining nature (biomimicry [8]), the equilibrium design approach (simulated annealing [9]) - seeking lowest energy level to achieve the design.

\section{Formulating the design problem}

The traditional approach to addressing a design problem is to break down the problem into functional requirements (FRs), or design 'needs', and Design Parameters (DPs), or engineering specifications. FRs are usually qualitative and DPs must be quantitative. Examples for which design parameters could be based are: Quality - reliability, robustness, efficiency, appearance, safety, productivity; Engineering - geometric, weight, strength, dynamic, thermodynamic, stress, heat transfer, wear, power; Human - ethics, aesthetics, prestige, preferences, feelings, five senses.

To insure that FRs are being achieved, and to provide a quantitative measure of how well the design solution meets the needs, FRs are mapped to one or more of the DPs. In recognizing that good design requires equal emphasis on the three design activities of Quality, Economics, and Requirements, it is natural to conclude that there may be dependence between a functional requirement and one or more design parameters. Indeed, the three design activities can have conflicts, where improvements to one parameter may negatively influence another, such as volume versus weight.

To provide a better measure of how DPs affect each FR, a matrix associating the weight each DP influences each FR can be formulated as shown in Equation 1. In other words, each row of the matrix associates an FR to a weighted linear sum of the DPs.

$$
\{F R s\}=[A]\{D P s\}
$$

The underlying concept of determining the numerical values for the matrix is based on the "House of Quality" [10] used in marketing, where FRs and DPs are formed and users come up with the quantitative values. By having the numerical values in the matrix, mathematical analysis can be performed, especially utilizing the tools developed for statistical analysis of multivariate systems.

Through a weighted matrix, the integrated design methodology provides a further step to aid the development of better designs by permitting an analysis of the weighted matrix to identify the parameters that most affect the overall design. Through this, one can achieve the minimum number of independent parameters to satisfy the design goals. In addition, the design process should also be able to achieve inverse relationships between the functional requirements and design parameters. Imbedded in this process is a design analysis step where design redundancy, robustness, and conflicts can be evaluated and addressed.

The process of developing an associative matrix between the FRs and the DPs is not unique. It is a method used by Suh [11] under the Axiomatic Design methodology to evaluate if a design is 'good', however in Suh's application, the matrix is solely associative and weights are not given. Suh is using the matrix as a tool to organize the FRs and DPs in order to test his axioms. One of the difficulties associated with the Axiomatic Design process is that the method does not provide a means to uncouple functional requirements 
and design parameters. Suh suggests that through iterative processes, the association matrix can be either decoupled or uncoupled by reformulating the problem. This iterative process can be a "somewhat nebulous and protracted affair" [12], and therefore benefits from a methodology that can minimize the number of iterations.

\subsection{Quality of a design}

As a tool to address the Quality aspect of design, we take from Axiomatic Design its definition of what is considered 'good', that is, the independence and information axioms. A good design occurs when the FRs are independent of each other (i.e. uncoupled or decoupled matrix, and likewise 'bad' when the matrix is coupled). A good design also has minimum information content, that is, less information or complexity implies a more robust design (similar to Taguchi's approach to robustness in quality). This axiomatic design analysis is especially important in the early stages of the design, however, as noted above, lacks good tools to determine the minimum number of independent parameters to satisfy the design goals, especially if one assumes there are correlations between the three design activities.

\subsection{Economics and Requirements of a design}

Defining the functional requirements and design parameters for the design activities of Economics and Requirements is generally straight forward for many parameters. There are a broad range of tools available from business and engineering to provide quantitative measures of performance. These tools can be subjective, e.g. preference ranking on colors, statistical, e.g. surveys on quality perception, or analytical, e.g. return on investment or stress calculations. Aesthetic measurement is more difficult. Although it can addressed through subjective tools such as ranking and surveys,

Table 1. Functional Requirements of the Desk Lamp

\begin{tabular}{|c|c|c|}
\hline 1 & Appropriate reach or envelope & R,Q \\
\hline 2 & Good lighting effectiveness & R,Q \\
\hline 3 & Compact mechanism & R \\
\hline 4 & Easy to use & $\mathrm{Q}$ \\
\hline 5 & Stable & $\mathrm{R}$ \\
\hline 6 & Safe & $\mathrm{R}$ \\
\hline 7 & Low cost & $\mathrm{E}$ \\
\hline 8 & Aesthetically pleasing & $\mathrm{R}$ \\
\hline 9 & Efficient & $\mathrm{R}, \mathrm{E}$ \\
\hline 10 & Low heat generation & $\mathrm{R}, \mathrm{E}$ \\
\hline \multicolumn{3}{|c}{ Q-Quality, E-Economics, R-Requirements } \\
\hline
\end{tabular}


Table 3. Weighting Matrix for Desk Lamp Design

\begin{tabular}{|c|c|c|c|c|c|c|c|c|c|c|c|c|c|}
\hline & \multicolumn{12}{|c|}{ Design Parameter } \\
\hline & & 1 & 2 & 3 & 4 & 5 & 6 & 7 & 8 & 9 & 10 & 11 & 12 \\
\hline \multirow{10}{*}{ 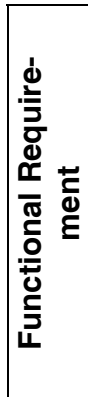 } & 1 & 0.10 & 0.93 & 0.60 & 0.53 & 0.47 & 0.20 & 0.40 & 0.40 & 0.20 & 0.03 & 0.60 & 0 \\
\hline & 2 & 0.97 & 0.67 & 0.07 & 0.13 & 0.23 & 0 & & 02 & 010 & 0.73 & 0.10 & 0.27 \\
\hline & 3 & 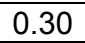 & & & & 0. & & & & & 0.0 & & 0 \\
\hline & 4 & 07 & 0.57 & 0. & 0.63 & 0.67 & & .1. & .43 & 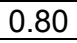 & 0.80 & 0.40 & 0.23 \\
\hline & 5 & 10 & 0. & 0.6 & .80 & 0.50 & & 0.4 & 0.4 & .53 & 0 & 1 & 0 \\
\hline & 6 & 0.70 & 0.43 & 0.17 & 0.40 & 0.30 & 0. & 0.2 & 0.30 & 0.50 & 0.3 & 0.80 & 0.97 \\
\hline & 7 & & 0.53 & 0.4 & 0.50 & 0 & & 0.8 & 0.5 & 0.17 & 0.6 & 0.27 & 0.33 \\
\hline & 8 & 0.00 & & 0.4 & & & & & 0.9 & 0 & 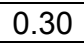 & .60 & 0 \\
\hline & 9 & 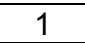 & 0.53 & 0.27 & 0.40 & 0.37 & 0. & 0.5 & 0.17 & 0.33 & 0.70 & 0.30 & 0.60 \\
\hline & 10 & 1 & 0.43 & 0 & 0.10 & 0.10 & 0.40 & 0.10 & 0.30 & 0 & 0.63 & 0 & 0.93 \\
\hline
\end{tabular}

Table 3 shows one weighting matrix that was developed based on an averaged opinion of those weights. Note that the weights within the matrix have been normalized between zero and one. This is because it is convenient to think of these weights as being similar to a correlation coefficient between two statistical variables. Tools, such as the analytic hierarchy process [15], can be used to formulate this matrix if a more systematic approach is needed.

\section{Analysis of the weighting matrix}

The advantage of framing the relationship between the functional requirements and the design parameters as a weighted matrix is readily apparent when one considers the many tools that are available for evaluating matrices. These include rank analysis, eigenvector analysis and their application to tools developed for data analysis such as principal component analysis, cluster analysis and multi-dimensional scaling, and factor analysis.

\subsection{Rank analysis}

A simple analysis of the relationship between the functional requirements is to test if the weighting matrix has full rank. If the rank of the matrix is not full, this tells us that the matrix has redundant information, and the redundant information needs to be eliminated. Matrix analysis program such as Matlab ${ }^{\circledR}$ can be used to assess the rank, and allow a precision to be placed on this assessment through examining the singular values of the matrix to some level of tolerance.

To interpret rank evaluation in terms of design, if an FR has an identical set of weightings on the DPs as another FR, it is likely to be characterizing the same need, and may be a semantic error. An examination of the functional requirement definitions is in order, with the appropriate redefinitions and removal of rows to achieve full rank. One must be careful with the size of the tolerance in this analysis, as it is dependent on the values in the matrix. For example, the identity matrix, which is full rank, will show zero rank when the tolerance is set to 1 .

\subsection{Orthogonal factors}

For greater insight into the relationship between FR to FR or DP to DP, the weighting matrix may be transformed into a square 'correlation' matrix. Consider each row vector as an $n$-dimensional measure of the DPs on each FR. The degree to which an FR (row) is coupled to another FR (row) can be measured by its orthogonality. This is readily calculated from the cosine of their angle by taking the Euclidean inner product of the two vectors

$$
\cos \theta=\frac{\mathbf{u} \cdot \mathbf{v}}{\|\mathbf{u}\|\|\mathbf{v}\|} .
$$

If the cosine is zero, the vectors are orthogonal, or uncoupled, if it is one, the vectors are parallel, or completely dependent, giving a numerical measure of coupling between the two vectors. Thus an upper diagonal (or symmetric square) matrix can be formed, where each element represents the degree of coupling between a pair-wise comparison of FRs as measured through their weighting on the DPs. Likewise, each column vector of the weighting matrix can be evaluated to create a 'correlation' matrix showing the degree of coupling between the DPs as measured through their weighting on the FRs.

\subsection{Principal component analysis and cluster analysis}

In the design process, designers often come up with several FRs and DPs without knowing the importance of the individual parameters or their independence. Through principal component analysis (PCA) between FRs and DPs, designers can identify the critical parameters by minimizing information to satisfy 


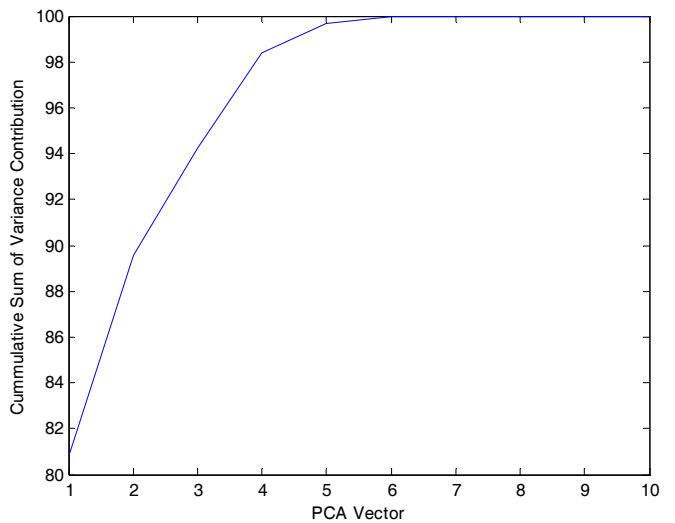

Figure 3. Information contribution of principal component analysis vectors

the good design requirements. PCA has been investigated in design for screening design variables [16].

Principal component analysis (PCA) is a tool used to explain the variance-covariance structure of a set of variables. It involves an eigenvector analysis of a matrix wherein a majority of the total system variability can be accounted for with a smaller number of principal components. Cluster analysis is a process of using certain measures of distance for grouping objects. It is useful as an informal means of determining interesting relationships between factors [17].

Since the weighting matrix is developed based on expert opinion and an a priori examination of the design problem, its elements are expected to contain noise. This noise can be minimized by conducting a PCA, allowing a manipulation of only the most relevant contributions. It has been found through experience that the orthogonal factors matrices provide the

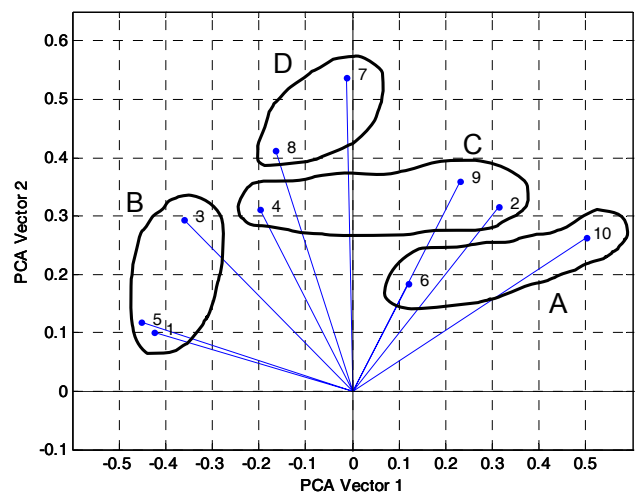

Figure 4. Grouped FRs by PCA and K-means

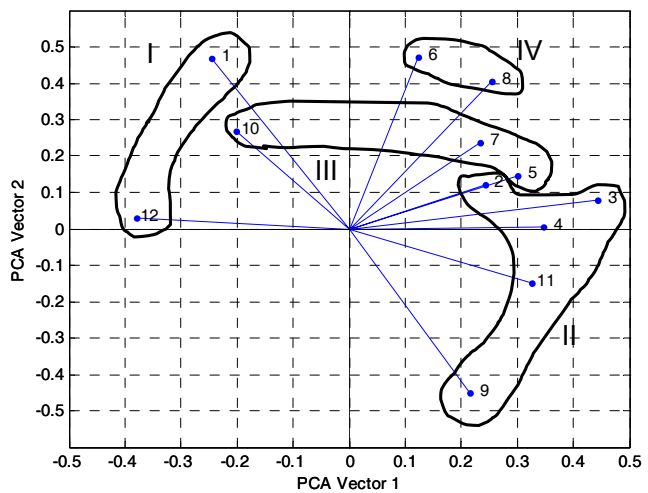
Figure 5. Grouped DPs by PCA and
K-means

best discrimination for the PCA analysis. The first step of the PCA analysis is to determine the eigenvalues and vectors of the matrix.

The ratio of each eigenvalue to the sum total of all eigenvalues is a measure of the contribution each eigenvector has as a principal component. The cumulative sum of these contributions gives a measure of the amount of information retained by the reduced eigenvector model. One model to determine the number of eigenvectors to retain is to set a required 'level' of information to retain. If we wish to retain $95 \%$ of the information, the cumulative sum plot of the eigenvalues, Figure 3, indicates four eigenvectors.

The retained eigenvectors can then be used in a cluster analysis, in conjunction with engineering judgment, to determine which design factors are strongly connected. One cluster analysis tool is the $K$ means method. Briefly, a set of data (vectors) is partitioned into $k$ clusters by iteratively assigning items to clusters and calculating the Euclidean distance between the centroids of the items, until the distance between the cluster centroids is maximum relative to the distance within each cluster to its centroid [17].

Engineering judgment should be used to determine the number of clusters and the logic of a factor's membership in a given cluster. Figure 4 displays a 2dimensional biplot of the first two principal components of the FRs, with the factors grouped into four clusters based on the four eigenvectors determined in the PCA. Figure 5 displays a two dimensional biplot of the first two principal components for the DPs, grouped into four clusters based on four PCA eigenvectors. Table 4 and 5 summarizes these groupings, providing new labels for each super-set to describe what design parameters it represents.

The benefit of the $K$-means clustering method is its predefined number of groups, allowing the forma- 
Table 4. Grouped FRs

\begin{tabular}{|c|c|c|}
\hline A & 6,10 & Safe to Operate \\
\hline B & $1,3,5$ & Efficient in Space \\
\hline C & $2,4,9$ & Efficient to Use \\
\hline D & 7,8 & Consumer Appeal \\
\hline
\end{tabular}

Table 5. Grouped DPs

\begin{tabular}{|c|c|c|}
\hline I & 1,12 & Luminosity and Temperature \\
\hline II & $2,3,4,9,11$ & Mechanism Size \\
\hline III & $5,7,10$ & Mechanism Complexity \\
\hline IV & 6,8 & Aesthetic Measures \\
\hline
\end{tabular}

tion of a square 'super-set' weighting matrix. Table 6 rearranges the rows and columns of Table 3 based on the PCA and cluster analysis grouping of similar factors. Table 7 summarizes the grouped factors by averaging the individual weights to give a measure of weight for the 'super-set' factor. As can be seen by examining this new weighting matrix, the grouped factors are more uncoupled. The designer can then take each super-set of FRs and DPs, and knowing their dependence, can either redefine the included factors to simplify the design, or develop the detailed design with this dependence and potential conflicts in mind. For example, in the new DP grouped factor, 'Mechanism Complexity', the design parameters 'Number of Joints' and 'Number of Components' could be redefined as 'Number of Moving Elements'.

\subsection{Multi-dimensional scaling analysis of DP and FR orthogonal factors}

Additional insight of the relationship within DPs and FRs can be obtained from a multi-dimensional

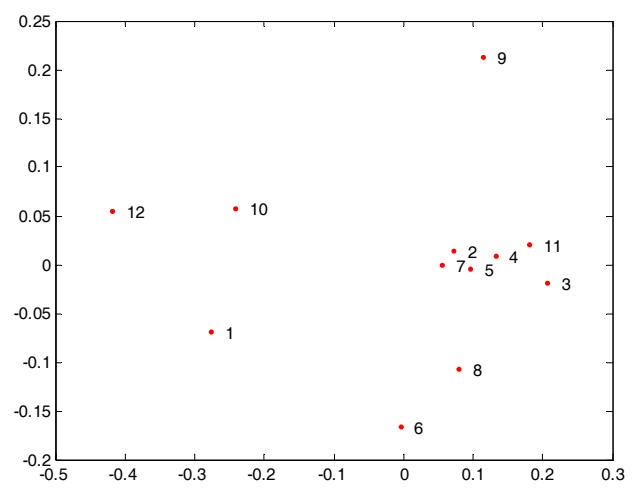

Figure 5. Multi-dimensional scaling of
orthogonal DP vectors.

scaling analysis of the orthogonal factors matrices. Multi-dimensional scaling is another clustering method for transforming multivariate data into lowdimensional space, allowing the design to better visualize the relationship between variables. The technique seeks "to find a representation of items in few dimensions that the inter-item proximities 'nearly match' the original similarities" [17]. Multi-dimensional scaling is used extensively in marketing to understand the relationships between dissimilar product descriptors, e.g. [18]. Therefore, by examining the distance between points on a multi-dimensionally scaled plot of the orthogonality of DP or FR vectors, the designer can get an idea of how factors are similar, with closer points being more similar. Figure 5 shows the relationship between DPs for the desk lamp. This plot shows that DPs 2,4,5,7 and 11 (extension length, fixed or free base, number of joints, number of components, and

Table 6. PCA Weighting Matrix for Desk Lamp Design (matrix $A^{*}$ )

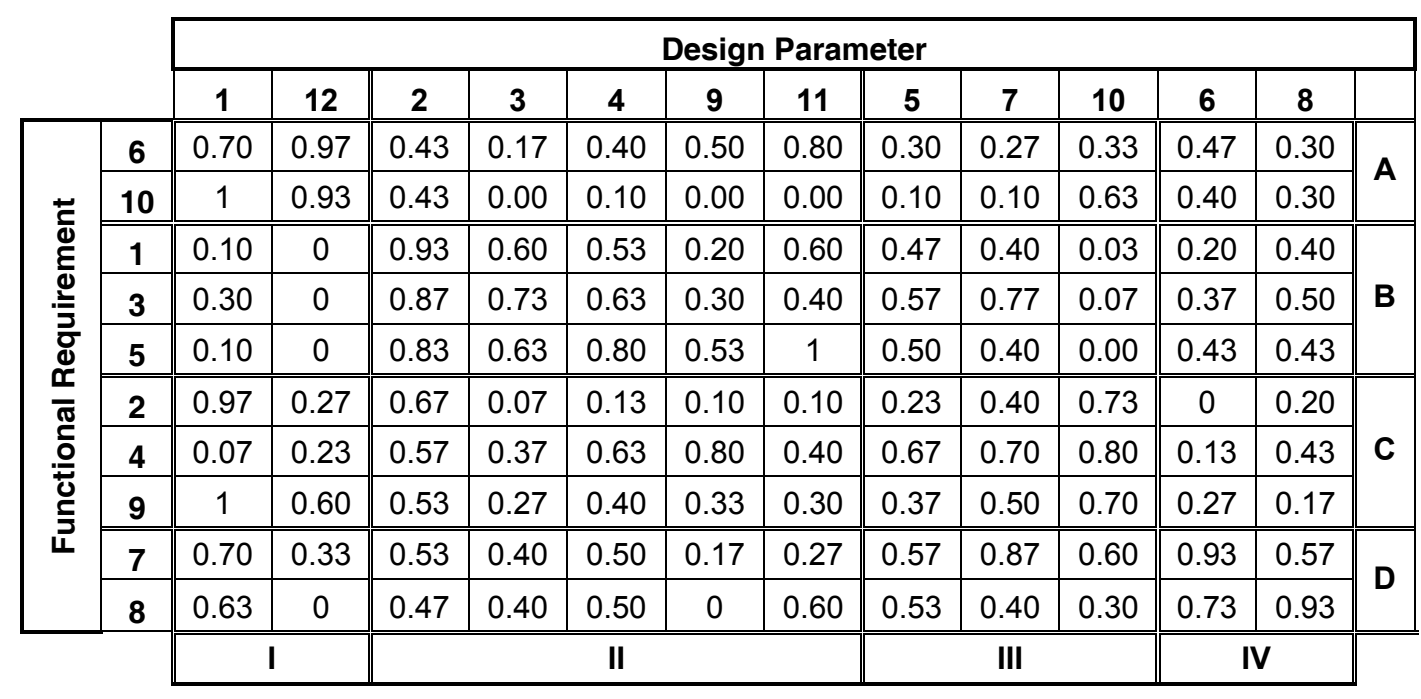


Table 7. New PCA Weighting Matrix for Desk Lamp Design

\begin{tabular}{|c|c|c|c|c|c|}
\hline & \multicolumn{4}{|c|}{ DP } \\
\hline & & 1 & II & III & IV \\
\hline \multirow{4}{*}{ FR } & $A$ & 0.90 & 0.28 & 0.29 & 0.37 \\
\hline & B & 0.08 & 0.64 & 0.36 & 0.39 \\
\hline & $C$ & 0.52 & 0.38 & 0.57 & 0.20 \\
\hline & $D$ & 0.42 & 0.38 & 0.54 & 0.79 \\
\hline
\end{tabular}

center of gravity) have similar influence on a set of (non-specified) FRs, while 3 and 12 (foot print of lamp and temperature of component) influence different FRs.

\subsection{Backward analysis}

The Axiomatic Design process has been criticized due to its inability to perform backward analysis [19]. By providing numerical weights to the weighting matrix and perform the independence analysis through the PCA, we can also perform backward analysis by inverting the new simplified weighting matrix (Table 7) as shown in Table 8. The negative values in the inverse matrix represent adverse effects on the DPs by FRs. High positive values (shown as the diagonal values) also indicate the uncoupling between DPs and FRs. The investigation of backward analysis also aid designers to come up with better FRs and DPs through iterative processes to achieve desired goals.

\section{Conflict resolution and fine tuning}

The conflict between the three design activities can be resolved through minimizing the coupling of the weighting matrix. Often, coupling between FRs and DPs is inevitable. When this is the case, other conflict resolution methods such as rule based Fuzzy analysis can be utilized to find the optimum solution based on a given set of rules.

\section{Conclusion}

Integrated design consists of three related factors, economics, quality, and requirements. The design process should analyze all three factors in order to achieve exceptional, competitive designs.

An extension of the axiomatic design concept is presented by providing weighting factors between the FRs and DPs similar to the house of quality. These weighting factors can be obtained by both qualitative and quantitative means. In our case, we have performed qualitative analysis by averaging three weighting factors from design engineers.
Table 8. Inverse Weighting Matrix for Desk Lamp Design

\begin{tabular}{|c|c|c|c|c|c|}
\hline & \multicolumn{4}{|c|}{ FR } \\
\hline & & $A$ & $B$ & C & $D$ \\
\hline \multirow{4}{*}{ DP } & 1 & 1.40 & -0.29 & -0.06 & -0.49 \\
\hline & II & 0.74 & 2.39 & -0.55 & -1.37 \\
\hline & III & -1.84 & -1.28 & 2.75 & 0.79 \\
\hline & IV & 0.17 & -0.12 & -1.59 & 1.65 \\
\hline
\end{tabular}

By performing a principle component analysis, one can find the important factors affecting FRs and DPs and can group similar factors to reduce the number of FRs and DPs. This simplifies designs and aids designers to come up with better design concepts based on the underlying FRs and DPs.

A method of backward analysis has been developed. In order for a backwards analysis concept to work, the backward operator needs to make sense. The inversed weighting matrix provides a better understanding between DPs and FRs and negative values represent adverse effects on the DPs by FRs.

This work provides a new way of looking at design methodologies by integrating known methodologies (i.e. axiomatic design and house of quality) to provide more optimal designs. Further study is needed to investigate more examples and verify the effectiveness of the proposed methodology, as well as measure its 'cycle time' and test its ability to facilitate a rapid design process. In addition, ways to minimize design iterations should be investigated.

\section{Acknowledgements}

The authors wish to thank the Department of Mechanical and Manufacturing Engineering and the Faculty of Engineering at the University of Calgary for their support of this research. Thanks also to Dr. P. Gu for invaluable discussion of design methodologies.

\section{References}

[1] Dixon, J.R., Design Engineering: Inventiveness, analysis, and decision making, McGraw Hill, 1982.

[2] Seliger, G., Buchholz, A., "Product Innovation - Industrial Approach", Annals of CIRP, 50(2), 2001, p.425.

[3] Van Brussel, H., "Mechatronics - A powerful concurrent engineering framework", IEEE/ASME Mechatronics, 1 (2), 1996, pp. 127-135.

[4] Gu, P., Hashemian, M., Nee, A.Y.C., "Adaptable Design", Annals of CIRP, 53(2), 2004.

[5] Westkamper, E., Alting, L, Arndt, G., "Life Cycle Management and Assessment: Approaches and visions towards sustainable manufacturing", Annals of CIRP, 49(2), 2000, pp. 501-522. 
[6] Peters, T., The Circle of Innovation, Vintage Press, 1999.

[7] Altshuller, G., Innovation Algorithm, Technical Innovation Center, 1999.

[8] French, M., Invention and Evolution, Cambridge University Press, 1994.

[9] Besnard, E., Cordier-Lallouet, N., Kural, O., Cehn, H., "Design/Optimization with Advanced Simulated Annealing", AIAA, 1999-0186.

[10] Hauser, J. R.., Clausing, D., The House of Quality, Harvard University Press, 1988.

[11] Suh, N., Principles of Design, Oxford University Press, 1990.

[12] Mann, D., “Axiomatic Design And TRIZ: Compatibilities and Contradictions", TRIZ Journal, June, 1999, http://www.triz-journal.com.

[13] Birkoff, D., Aesthetic measure, Cambridge Press, Massachusetts, 1933.

[14] Rudolph, S., A Methodology for the Systematic Evaluation of Engineering Design Objects, PhD Thesis, Institute of Statics and Dynamics of Aerospace Structures, Stuttgart University, 1995.

[15] Saaty, T., The Analytic Hierarchy Process, McGrawHill, New York, 1980.

[16] Han, S. H., Kim, J., "A comparison of screening methods: Selecting important design variables for modeling product usability", International Journal of Industrial Ergonomics, 32, 2003, pp. 189-198.

[17] Johnson, R.A., Wichern, D.W., Applied Multivariate Statistical Analysis, Prentice Hall, 2002.

[18] Petiot, J-F., Yannou, B., "Measuring consumer perceptions for a better comprehension, specification and assessment of product semantics", International Journal of Industrial Ergonomics, 33, 2004, pp. 507-525.

[19] Alamaro, M., "Voodoo Engineering", Machine Design, July 2004. 